\title{
Bronchial challenge with flour: early response is dependent on the dose of activated allergen inhaled
}

\author{
D. Choudat*, J.F. Fabriès", J.C. Martin*, C. Villette*
}

Bronchial challenge with flour: early response is dependent on the dose of activated allergen inhaled. D. Choudat, J.F. Fabriès, J.C. Martin, C. Villette. C ERS Journals Ltd 2002

ABSTRACT: Specific bronchial challenges provide information about the relationship between inhaled dose of allergen and change in lung function, but the intermediate pathways remain largely obscure. The aim of this study was to investigate the relationships between the early asthmatic response and 1) the inhaled dose of wheat flour, 2) the concentration of wheat flour, 3) the duration of the exposure, and 4) the deactivation of inhaled allergens and mediators.

Thirty-one patients with occupational asthma to wheat flour were studied. Particle aerosols were generated by a computer-controlled aerosoliser and the results were expressed as the provocative dose causing a $20 \%$ fall in forced expiratory volume in one second (FEV1) (PD20). The cumulative dose (from the beginning of the challenge), the last inhaled dose, and an estimated dose (taking into account exponential deactivation), were calculated.

Twenty patients had high reactivity to flour (reaching a PD20 value). Eleven patients had intermediate reactivity (no measurable PD20 but significantly greater fall in FEV1 compared with lactose challenge). A better correlation between change in FEV1 and dose was obtained for the estimated dose than for the cumulative or last inhaled dose.

The bronchial response to wheat flour can be measured by the individual specific hyperreactivity and is expressed by provocative dose of flour. However, deactivation of the allergen and mediators has to be taken into account. This problem can be addressed by using a mathematical model.

Eur Respir J 2002; 20: 409-416.
*Cochin University Hospital, AP-HPUniversité Paris 5, Paris, and "\#rench National Research and Safety Institute (INRS), Vandoeuvre, France.

Correspondence: D. Choudat, Hôpital Cochin, Service de Pathologie Professionnelle, 27 rue du Faubourg SaintJacques, 75014 Paris, France.

Fax: 33158412794

E-mail: dominique.choudat@ech. ap-hop-paris.fr

Keywords: Occupational asthma specific bronchial challenge wheat flour

Received: April 202001

Accepted after revision: October 18 2001

This study was supported by grants from Caisse Nationale d'Assurance Maladie des Travailleurs Salariés and Caisse Régionale d'Assurance Maladie d'Ile-de-France.
Specific bronchial challenges are used to confirm the diagnosis of occupational asthma and to measure specific reactivity $[1,2]$. The specific challenges are performed either in a small room, or with various aerosolisers. Sometimes the ambient concentration is measured, but inhaled dose is seldom determined. The way to express results and the criteria of positivity are not as well defined as for the methacholine test [1-4]. However, a $20 \%$ (or $15 \%$ ) fall in forced expiratory volume in one second (FEV1) is the criterion usually used to indicate a positive early reaction $[1,3-5]$. The current authors have demonstrated that the fall in FEV1 during the specific challenge can be compared to the distribution of the values of FEV1 during the control test with lactose [3]. If the inhaled dose is measured, the specific reactivity can be expressed as the provocative dose of flour causing a $20 \%$ or $15 \%$ fall in FEV 1 ( $\mathrm{PD}_{\mathrm{f}} 20$ or $\mathrm{PD}_{\mathrm{f}} 15$, respectively). Several studies of various allergens have shown that the range of specific reactivity among asthmatic subjects is wide $[3,6-9]$.

The duration of the specific challenge tests varies according to: 1) the device and procedure employed; and 2) the bronchial reactivity of the subjects. The test is short when the FEV1 falls after the first inhalation of allergen, but in some cases the test can last several hours before a decrease in FEV1 is observed [1, 3]. VANDENPLAS et al. [7] have demonstrated that the main determinant of bronchial responsiveness to isocyanates, among subjects with a change in FEV $1>20 \%$, is the total inhaled dose and not concentration nor duration of exposure per se. Similar results have been found for other occupational [8] and environmental allergens since the work of TIFFENEAU [9] in 1958. However, the early change in FEV1 can disappear spontaneously within $\sim 1 \mathrm{~h}$ suggesting that the allergens and mediators are deactivated $[9,10]$. It is probably unnecessary to take this deactivation into account for challenges involving a quick increase of the inhaled doses and lasting $<1 \mathrm{~h}[6-8]$. In contrast, it is likely that the deactivation of allergens and mediators may decrease the early response if the test is extended for $>1 \mathrm{~h}$.

Specific challenge gives information about the inhaled dose of allergen and the change in FEV1 but, usually, no data about the intermediate steps. The current authors' working hypotheses were that: 1) the substances responsible for the early asthmatic response are deactivated according to an exponential model; and 2) there is a linear relationship between 
the dose of active mediators and allergen actually remaining in the bronchial airways and the change in FEV1.

Therefore, the aim of this study was to examine the relationships between the dose of wheat flour and the early response in FEV1 to assess the roles of: 1) the inhaled dose of allergen; 2) the concentration of the allergen; 3) the duration of exposure; and 4) the deactivation of inhaled allergens and mediators.

\section{Methods}

\section{Patients}

The study was approved by the local Ethics Committee, and written informed consent was obtained from each patient. Forty four patients referred for assessment of occupational asthma caused by flour agreed to undergo a specific bronchial challenge [3, 11]. All reported work-related symptoms of asthma and underwent a standard check-up including a questionnaire, spirometry, and methacholine test [4]. The challenges were performed with lactose and then with wheat flour, on two separate days. Bronchodilator and steroid treatments were stopped $24 \mathrm{~h}$ before the challenge. The subjects were outpatients and the challenges began at 09:00 $\mathrm{h}$.

Thirty-one subjects with significant change in FEV1 during the specific challenge were included to study the correlations between the inhaled dose and the bronchial response. From 21 subjects, whose data had been previously published [3], 14 were included in this study, and from 23 subjects tested more recently, 17 were included in the study group.

\section{Specific bronchial challenge}

Specific bronchial challenge was performed with an aerosol generator as described previously $[1,11]$. The equipment was fully computerised to calculate the dose of aerosol inhaled by the patient through a venturi system and a mouthpiece. The dose of wheat flour or lactose inhaled was calculated from the inspiratory flow rate and the instantaneous aerosol concentration measured by a calibrated photometer. The aerosol concentration inside the inhalation chamber was maintained close to $3.0 \pm 0.2 \mathrm{mg} \cdot \mathrm{m}^{-3}$ or to $5.0 \pm$ $0.2 \mathrm{mg} \cdot \mathrm{m}^{-3}$ (mean $\left.\pm \mathrm{SD}\right)$.

The patient was asked to breathe at tidal volume. Increasing the number and duration of periods of exposure increased the inhaled dose. The duration of the inhalation period was increased in a step-wise manner. A maximum of nine periods was used $(15 \mathrm{~s}$, $30 \mathrm{~s}, 1 \mathrm{~min}, 2 \mathrm{~min}, 5 \mathrm{~min}, 15 \mathrm{~min}, 20 \mathrm{~min}, 30 \mathrm{~min}$ and $30 \mathrm{~min}$ ). Therefore, the cumulative duration of exposure was $15 \mathrm{~s}$ to $104 \mathrm{~min}$, according to specific bronchial reactivity. Each period was separated from the next by an interval of $10 \mathrm{~min}$. During each inhalation period, the dose inhaled was continuously determined by integration of the instantaneous concentration and inspiratory flow rate. The cumulative inhaled dose from the beginning of the challenge was determined at the end of each period.

FEV1 was measured by flow/volume curves with an automated electronic spirometer 1 and $10 \mathrm{~min}$ after each lactose or flour inhalation period. The time/ response curve was immediately plotted by computer (fig. 1). The lower of the lung function values 1 or $10 \mathrm{~min}$ after each inhalation period and the cumulative dose from the beginning of the challenge were automatically plotted to establish dose/response curves. If there was $<20 \%$ change in FEV1, the patient was subjected to another inhalation period. The test was stopped once FEV1 decreased by $20 \%$ or after 104 min of exposure.

On the first day, a control test with lactose was performed. If lung function was stable, the flour challenge was performed on another day. Pharmacie Centrale (Assistance Publique, Hopitaux de Paris, France) provided the lactose powder and the wheat flour was a standard commercial product [11]. The mass median aerodynamic diameter of the particles inside the inhalation chamber was $\sim 6 \mu \mathrm{m}$ for wheat flour and $4 \mu \mathrm{m}$ for lactose.
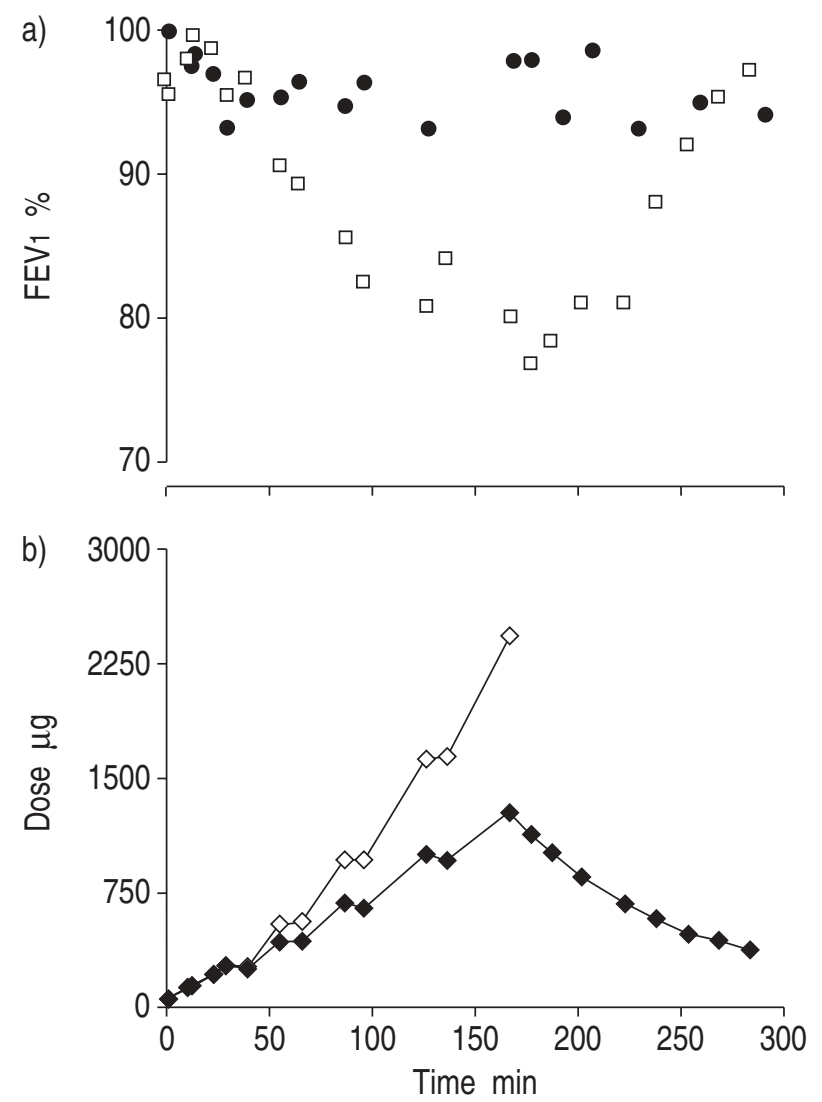

Fig. 1.-a) Time/response curves showing changes in forced expiratory volume in one second $\left(\mathrm{FEV}_{1}\right)$ over time during the test with lactose (@) and the test with wheat flour ( $\square$ ) for one subject. b) Time/dose curves showing the variation of the cumulative dose of flour $(\diamond)$ and the calculated dose $(\diamond)$ during the test with wheat flour taking into account deactivation (coefficient $\left.\mathrm{k}=0.014 \mathrm{~min}^{-1}\right)$. The periods of exposure, with increasing doses, are separated by periods for measuring $\mathrm{FEV}_{1}$. 
Expression and interpretation of the results of the specific challenge

Changes in lung function were evaluated according to the criterion of the American Thoracic Society for spirometry: if several maximal expiratory manoeuvres are performed, the FEV1 of the subject is the highest value recorded [12]. Therefore, the initial value was not used, but instead the highest FEV1 value recorded separately on each day (lactose or flour) was used, and the change in FEV1 at the end of each period of exposure calculated $[3,10]$.

The percentage of variation of the FEV1 during the tests with lactose or flour, the confidence interval limit of the percentage of variation of the FEV1 during the test with lactose, and the $\mathrm{PD}_{\mathrm{f}} 20$ and $\mathrm{PD}_{\mathrm{f}} 15$ during the test with flour were automatically calculated during the challenge and were used to define the level of the bronchial reactivity.

If FEV 1 decreased by $>15 \%$, the result of the specific bronchial challenge was expressed as the $\mathrm{PD}_{\mathrm{f}} 20$ or $\mathrm{PD}_{\mathrm{f}} 15$. The provocative dose was determined from the logarithm of the cumulative dose from the beginning of the challenge $\left(\mathrm{Q}_{\mathrm{c}}\right)$ as for the methacholine test [4].

Changes in FEV1 during the lactose test and the wheat flour test were also compared using confidence intervals (CI) [3]. For each patient, the percentage of changes in FEV1 during the lactose test were approximately consistent with a normal distribution. The mean and SD for changes in FEV1 during the lactose test for each patient were calculated to determine the lower $99.7 \%$ CI limit (mean- $(3 \times \mathrm{SD})$ ). The intermediate reactivity group included subjects with decreases in FEV1 that were $<20 \%$ but significantly different from that associated with the challenge with lactose (maximum decrease in FEV1 in the challenge with flour below the lower $99.7 \%$ CI limit of the control test).

Only subjects who had high or intermediate reactivity were included in this study.

\section{Expression of the dose}

Three expressions of the dose were used to establish dose-response relationships: 1) the cumulative dose from the beginning of the test $\left(\mathrm{Qc}_{\mathrm{c}}\right)$. The dose response was analysed as if all the inhaled flour was always bioavailable to induce a bronchoconstriction; 2) the dose inhaled during the last period of exposure $\left(\mathrm{Q}_{\mathrm{i}}\right)$; and 3) an estimated dose (Qe) calculated from the quantity of flour inhaled and taking into account some deactivation of the allergens contained in the flour.

The method for calculating $\mathrm{Qe}_{\mathrm{e}}$ is described in the appendix. The corresponding model for expressing $\mathrm{Qe}_{\mathrm{e}}$ as a function of time is not based on the mechanisms leading to bronchoconstriction. It is only a descriptive kinetic model that allows for the bioavailability of the allergens inhaled to be simply taken into account. For each subject, this model drew a family of dose/time curves according to the values of the kinetic constant (k) (fig. 1). These curves were located between the abcissa and $\mathrm{Q}_{\mathrm{c}}$; for the lower values of $\mathrm{k}$, the inhaled allergen was slowly deactivated, and the curves $\mathrm{Qe}$ were close to $\mathrm{Q}_{\mathrm{c}}$; for the higher values of $\mathrm{k}$, the inhaled allergen was quickly deactivated and the values of Qe were low, close to the abcissa. The dose/ response curves were then analysed according to the observed changes in FEV1 (fig. 2). The optimal value of $\mathrm{k}$ for each subject was determined from the best correlation between $\mathrm{Q}_{\mathrm{e}}$ and the variations of the FEV1 during the periods of exposure and those of recovery (fig. 3). The correlation coefficients and the optimal value of $\mathrm{k}$ were automatically calculated after each specific challenge. At the optimal value of $k$, the slope of the dose/response curve and the level of significance were also determined (fig. 4).

\section{Statistical analysis}

Descriptive statistics were used to summarise demographic characteristics of the patients. Logarithmic transformation was used for the data not normally distributed such as $\mathrm{PD}_{\mathrm{f}} 20, \mathrm{PD}_{\mathrm{f}} 15$ and optimal deactivation coefficient. Means and SD of the transformed data were calculated for the two groups and the results were expressed as geometric mean. An unpaired t-test was used to compare groups. A p-value $<0.05$ was considered as significant.

\section{Results}

The characteristics of two groups of subjects, defined according to specific reactivity, are described in table 1 .

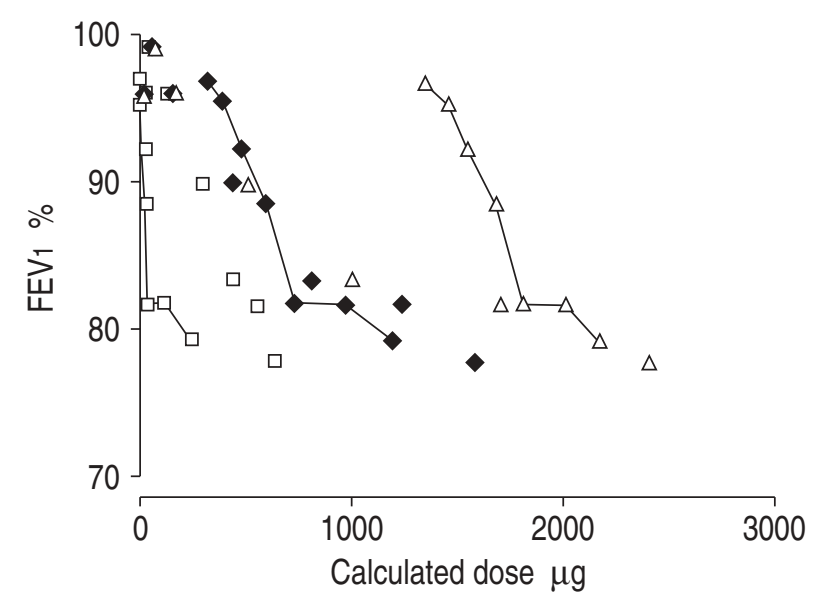

Fig. 2.-Dose/response curves for wheat flour challenge according to three values of the deactivation coefficient $(\mathrm{k})$ for the subject illustrated in figure 1. Higher values of $\mathrm{k}$ correspond to the inhaled allergen being quickly deactivated during the periods of exposure ( $\square$ ); after the end of exposure the estimated dose decreases quickly while the forced expiratory volume in one second (FEV1) remains impaired ( $\left.-\square-; 0.05 \mathrm{~min}^{-1}\right)$. The lower values of $\mathrm{k}$ correspond to the inhaled allergen being slowly deactivated: the dose/response curve becomes parallel to the abcissa with increasing dose $(\triangle)$, and the estimated dose remains high while the FEV 1 normalises $\left(-\triangle-; 0.005 \mathrm{~min}^{-1}\right)$. The optimal value of $\mathrm{k}\left(0.014 \mathrm{~min}^{-1}\right)$ was determined as that giving the best correlation between the dose and the FEV 1 during the periods of exposure $(\bullet)$ and of recovery $(-\bullet-)$. 


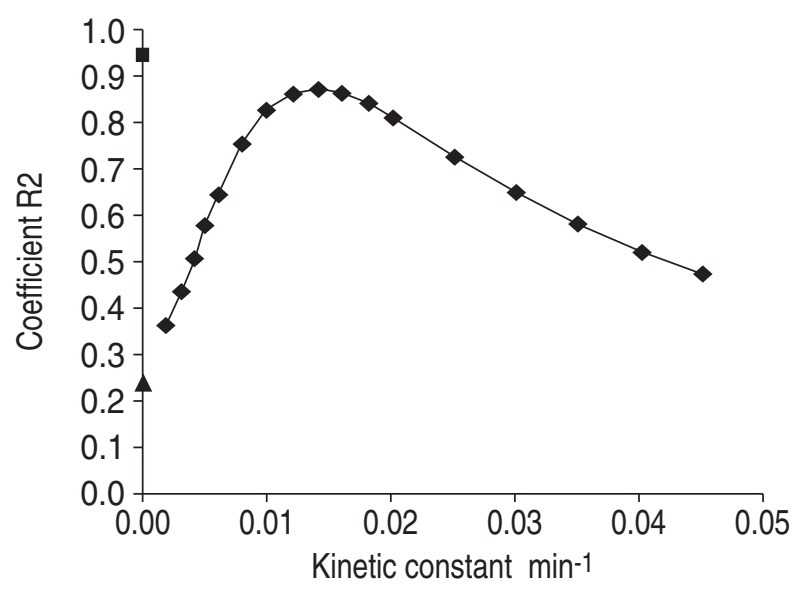

Fig. 3.-Correlation coefficients for cumulative dose ( $\boldsymbol{\Delta})$, last inhaled dose ( $\boldsymbol{\square})$ and calculated doses according to the deactivation coefficient $(--)$. The higher correlation coefficient between the calculated doses and the variations of the forced expiratory volume in one second during the periods of exposure and of recovery determined the optimal value of the deactivation coefficient for this subject $\left(\mathrm{k}=0.014 \mathrm{~min}^{-1}\right)$. Cumulative dose and last inhaled dose are plotted for $\mathrm{k}=0$.

The inhalation of lactose did not significantly affect the FEV1 of the patients. The cumulative dose inhaled was $2,106 \pm 851 \mu \mathrm{g}$ (mean \pm SD). Slight changes in FEV1 that did not depend on the dose inhaled were observed. These changes were consistent with the typical reproducibility of FEV1 determinations.

The total amount of flour inhaled by each subject depended on the subject's specific hyperreactivity (table 1). The patients were assigned to one of two groups of specific reactivity. 1) Twenty subjects were included in the high reactivity group. The challenge with flour was clearly positive with a fall in FEV1 of $>20 \%$. The decrease in FEV1 was significantly

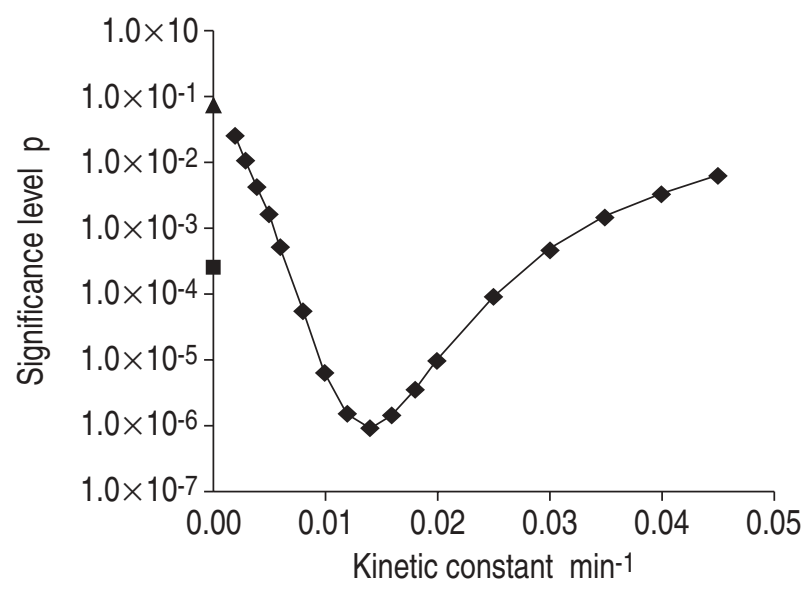

Fig. 4.-Significance of the correlations between forced expiratory volume in one second variations and cumulative dose $(\boldsymbol{\Delta})$, last inhaled dose ( $\boldsymbol{\square})$ and calculated doses according to the deactivation coefficient (- - The optimal value of the deactivation coefficient was associated with the greatest significance. Cumulative dose and last inhaled dose are plotted for $\mathrm{k}=0$.

different from the distribution of the FEV1 observed during the test with lactose. The maximum decrease in airflow was observed $\sim 10$ min after the last inhalation period. The duration of the challenge, from the beginning of the test to the end of the last inhalation period, was short (geometric mean $28 \mathrm{~min}$ ). Reversal of airway obstruction was spontaneous in the $20 \mathrm{sub}-$ jects within $1 \mathrm{~h}$ of the end of exposure. For each subject, the family of the dose/response curves was studied. The value of $\mathrm{k}$ was determined to obtain the best correlation coefficient between the estimated dose and the change in FEV1 observed during the inhalation periods and after the end of the exposure. 2) Eleven subjects were included in the intermediate reactivity group. For each of these subjects, the change in FEV1

Table 1.-Characteristics of the subjects according to specific bronchial reactivity to wheat flour and results of the challenges with lactose and flour

\begin{tabular}{|c|c|c|}
\hline & High reactivity & Intermediate reactivity \\
\hline Subjects $n$ & 20 & 11 \\
\hline \multicolumn{3}{|l|}{ Characteristics } \\
\hline Sex M:F & $19: 1$ & 11:0 \\
\hline Age yrs mean $\pm S D$ & $32 \pm 9$ & $32 \pm 7$ \\
\hline Duration of work yrs mean \pm SD & $14 \pm 9$ & $14 \pm 8$ \\
\hline Methacholine challenge PD20 $\mu \mathrm{g}$ & $177(43-727)$ & $514(194-1360)$ \\
\hline Lactose challenge cumulative dose $\mu \mathrm{g}$ & $1824(1123-2964)$ & $2101(1374-3213)$ \\
\hline \multicolumn{3}{|l|}{ Wheat flour challenge } \\
\hline Cumulative duration of the exposures min & $8(2-37)$ & $86(54-137)$ \\
\hline \multicolumn{3}{|l|}{ Cumulative dose/response } \\
\hline Cumulative dose $\mu \mathrm{g}$ & $213(40-1117)$ & $2903(1447-5824)$ \\
\hline $\mathrm{PD}_{\mathrm{f}} 20 \mu \mathrm{g}$ & $118(20-690)$ & $\mathrm{NC}$ \\
\hline $\mathrm{PD}_{\mathrm{f}} 15 \mu \mathrm{g}$ & $81(14-455)$ & $1969^{\#}(843-4600)$ \\
\hline Last inhaled dose $\mu \mathrm{g}$ & $115(26-507)$ & $923(485-1758)$ \\
\hline \multicolumn{3}{|l|}{ Calculated dose/response } \\
\hline Optimal deactivation coefficient $\mathrm{min}^{-1}$ & $0.020(0.014-0.028)$ & $0.021(0.008-0.056)$ \\
\hline Slope $\mu \mathrm{g}^{-1}$ & $0.171(0.037-0.796)$ & $0.010(0.005-0.020)$ \\
\hline
\end{tabular}

Data are presented as geometric mean (range of SD) unless otherwise stated. M: male; F: female; PD20: provocative dose of methacholine causing a 20\% fall in forced expiratory volume in one second (FEV1); PDf20: provocative cumulative dose of flour causing a $20 \%$ fall in FEV1; NC: not calculatable; PDf15: provocative cumulative dose of flour causing a $15 \%$ fall in FEV1. "PDf15: calculated for seven subjects (four subjects had significant change but $<15 \%$ ). 
was $<20 \%$ but was significantly different from the distribution of FEV1 during the test with lactose. The duration of the challenge was long (geometric mean $166 \mathrm{~min}$ ). Time/response curves decreased slowly during exposure, then recovered (fig. 1). For each subject, the family of the dose/response curves was studied according to the values of $\mathrm{k}$ to determine the highest correlation coefficient (fig. 2). If the inhaled allergen was deactivated quickly (high values of $\mathrm{k}$ ), the maximal dose $\left(\mathrm{Qe}_{\mathrm{e}}\right)$ after the periods of exposure was low, the dose quickly approached zero while the FEV1 was still impaired. In contrast, if the inhaled allergen was deactivated slowly (low values of k), Qe was close to $\mathrm{Q}_{\mathrm{c}}$; in this situation, the dose/response curve became parallel to the abcissa with increasing length exposure, and after exposure Qe remained high while the FEV1 was normalising. The optimal value of $\mathrm{k}$ was also determined for each subject to obtain the best correlation between the estimated dose $\mathrm{Qe}$ and the variations of the FEV1 during the periods of exposure and of recovery (figs 2 and 3).

The mean values of the coefficients of deactivation were not significantly different between the two groups (table 1). For each subject, the significant levels of the correlations between the doses and the FEV1 variation were higher for $Q_{e}$ than for $Q_{c}$ or $Q_{i}$ (fig. 4).

\section{Discussion}

The relationship between the dose of flour inhaled and the early bronchial response was investigated. The late asthmatic response was not addressed because the mechanisms of the early and late response are different, so their relationships with the inhaled dose of allergen should be established separately. The subjects studied displayed a wide range of specific reactivity for wheat flour, as generally observed for other environmental or occupational allergens. The early response was related both to the individual's specific reactivity and to the inhaled dose. The individual's specific reactivity may be related to nonspecific bronchial and specific immunological reactivities [3, 9]. However, for a model to describe the response, it was also necessary to take into account deactivation of the inhaled allergen during the specific challenge test. The coefficient of deactivation was determined for each asthmatic subject. The range of the coefficient of deactivation was small, and without significant difference between the subjects with high and moderate specific hyperreactivity.

Specific bronchial challenges are used to confirm the diagnosis of occupational asthma $[1,2]$. However, the expression and interpretation of their results are not as well defined as for the methacholine test [4]. The authors have demonstrated that both $\mathrm{PD}_{\mathrm{f}} 20$ and $\mathrm{PD}_{\mathrm{f}} 15$ can be used to express the result and to compare with the lactose control test [3]. Nevertheless, the most appropriate inhaled dose must be determined for dose/ response curves to be established.

Firstly, Qc was used from the beginning of the challenge to determine $\mathrm{PD}_{\mathrm{f}} 15$ and $\mathrm{PD}_{\mathrm{f}} 20$. This method was suitable for patients with high hyperreactivity, because the initial periods of exposure were short and each additional dose caused an additional effect [3]. As for isocyanate, the main factor for bronchoconstriction was probably the inhaled dose, rather than the concentration or the duration of the exposure [7, 8]. However, for patients with low reactivity, the periods of exposure were long and, thus, the effect of the first doses may have been small or nonexistent. In such cases, changes in FEV1 may be largely a consequence of the last inhaled dose or, more precisely, the dose remaining in the lung taking into account the deactivation of the flour allergens and mediators [10, 13]. The authors constructed a model to take into account this deactivation and calculate Qe. Moreover, $\mathrm{PD}_{\mathrm{f}} 20$ and $\mathrm{PD}_{\mathrm{f}} 15$ could be calculated from $\mathrm{Qe}$ instead of from Qc or Qi.

The respective effects of concentration, duration of exposure and inhaled dose of various allergens have been studied. Most of these studies included subjects with high specific reactivity and the challenges lasted $<1 \mathrm{~h}$. Therefore, the impact of deactivation on the early asthmatic response was probably small, and possibly insignificant. Moreover, comparison of data between studies is difficult because of the diversity of methods used for the specific challenge and as the duration of exposure is not specified in some of the reports. The methods of specific challenge differ in the nature and the physical state of the allergens (gas, dry aerosol, aqueous extract), in the delivery procedure, and in the protocol for increasing the dose. There are two major types of protocol for determining dose/response relationships: 1) increasing the time of exposure at controlled concentration with closed-circuit devices [1-3,9]; and 2) geometrically increasing the concentration or the dose of the allergen nebulised from a dosimeter and inhaled for $1-5$ breaths $[6,14,15]$.

In 1958, TIFFEnEAU [9] demonstrated that the ventilatory response to house dust among 50 asthmatic patients was related to several factors: 1) the level of the individual's "allergie pulmonaire" (lung allergy) that controlled the production of mediators; 2) the deactivation of the mediators during continuous challenge; and 3) the level of the nonspecific bronchial "hyperrexcitability". It was shown that an equilibrium between anabolism and catabolism of mediators was reached after 15-30 min of continuous exposure. The quantity of mediators was constant thereafter. Finally, it was shown that this quantity triggered the bronchoconstriction and determined its intensity. The present study, thus, confirms the findings of TiffeneAu [9]. Furthermore, the present study describes and defines the inhaled doses of allergens and proposes a mathematical model taking inhalation and deactivation into account.

The effects of the concentration of isocyanates and of the duration of exposure during challenge on four asthmatic workers were studied by VANDENPLAS et al. [7]. For a given subject, the early reaction depended mainly on the inhaled dose. However, the true inhaled dose was not measured because the concentration, but not the inspiratory volume, was controlled. The four subjects were challenged with different concentrations of isocyanate and with different durations 
of exposure, such that the total dose (concentration $\times$ duration of exposure) was the same in all cases. Three subjects had high reactivity. Their challenges lasted $<12 \mathrm{~min}$. One subject was exposed for between 4-90 min (high and low concentrations, respectively). The exposures at high concentrations and low durations (cumulative doses from 321-404 parts per billion $(\mathrm{ppb})$ ) induced an immediate response. In contrast, similar cumulative doses $(274,317$ and $420 \mathrm{ppb})$ at lower concentration with longer durations $(45,68$ and $90 \mathrm{~min}$, respectively) did not induce an early asthmatic response. This difference between short and long exposures, for this subject, may be explained by deactivation decreasing the early response when the duration of exposure was long enough, or by decreasing ventilation during the longer exposures, causing a decrease in the actual inhaled dose.

The determinant of the bronchial response has also been studied for high molecular weight occupational agents in a dry aerosol form [8]. Seven subjects underwent two challenges with low and high concentrations of flour for various times to obtain similar change in FEV1. Two other patients were exposed to psyllium and garlic according to the same protocol. The authors concluded that the main determinant of the bronchial response was the inhaled dose and not the concentration or the duration. However, if the patients are classified according to the duration of exposure at low concentration, the results are suggestive of deactivation of the allergen. The patients with shorter durations at low concentration (2.2-8.5 min) had high reactivity. There were no significant differences between the two cumulative doses calculated at low and at high concentration for these patients. However, the patients with low reactivity had the longer durations of exposure (11, 15 and $120 \mathrm{~min}$ ). For this group, there were differences between the two cumulative doses; a higher inhaled dose was necessary at low concentration than at high concentration to obtain the same early asthmatic response. Therefore, these data also suggest deactivation of the allergen for longer exposures at low concentration.

CRIMI et al. [16] studied early asthmatic responses to an extract of Dermatophagoides pteronyssinus in 12 asthmatic subjects. On the first challenge, the subject inhaled 70 breaths at a given starting concentration. The dose was increased two-fold by doubling the allergen concentration until FEV1, measured 15 min after inhalation, fell below $80 \%$ of the baseline value. On the second challenge the highest concentration used on the first challenge was administered after control saline inhalation. The PD20 was calculated for the last inhaled concentration and not for the cumulative dose from the beginning of the challenge. The PD20 calculated from the challenges were very similar. The final cumulative dose was almost double the provoking dose inhaled on the second challenge, and the mean maximal fall in FEV1 tended to be higher (not significantly) with the increasing dose method than with the single dose method. These results are again consistent with allergen deactivation.

A few studies report no significant difference between an incremental dose of allergen and a bolus of the same total dose [6, 14]. TAYLOR et al. [6] compared incremental and bolus doses of allergen extract in a group of 35 asthmatic patients. Each concentration was administered by five breaths from a nebuliser. FEV1 was measured 5 and 10 min after administration of each concentration. Five times as much was administered if the FEV1 had not fallen by $>10 \%$. The specific reactivity of the subjects was relatively diverse, but the number of concentrations used for the challenges was only two to four. Therefore, the incremental challenges probably lasted $<1 \mathrm{~h}$. There was no significant difference in the fall of FEV1 after the incremental dose of allergen and the bolus of the same cumulative dose $(33.1 \pm 1.8$ versus $29.9 \pm 2.2 \%$, respectively). Probably, the last inhaled dose was the determinant for the effect and any deactivation was negligable for the early asthmatic response with this short protocol. However, deactivation was obvious because the mean change in FEV1 for the whole group was $10 \% 2 \mathrm{~h}$ after the challenge, and even two subjects whose FEV1 fell immediately by $\sim 60 \%$ recovered spontaneously to $20 \%$ within $10 \mathrm{~min}$.

Kumlin and Dahlin [14] also studied incremental and bolus doses of allergen extract (cat, dog, or grass) in a group of seven asthmatic patients with early reaction. For the incremental challenge, the doses were increased geometrically every $15 \mathrm{~min}$, until FEV1 had decreased by $\geqslant 20 \%$. The mean duration of the cumulative bronchoprovocation was $66 \pm 7 \mathrm{~min}$. The mean drop in FEV1 was not significantly different after the incremental dose of allergen and after the bolus of the same total dose $(29 \pm 2.4$ versus $25 \pm 2.8 \%$, respectively).

In sensitised subjects, there is a series of events following inhalation of allergens and before the change in FEV1: deposition of the allergens, binding to specific immunoglobulins E, activation of mast cells and basophils, release of the mediators, activation of the receptors, bronchoconstriction and finally the change in FEV1. The inhaled allergens [17] and the mediators are also deactivated [9]. This deactivation may be suspected from the recovery of lung function within $1 \mathrm{~h}$ of the end of the exposure. Therefore, the change in FEV1 may be related to the estimated dose remaining in the airways. In fact, the "estimated dose" of the allergen is dependent on the amount inhaled and amount deactivated, and also on the metabolism of the mediators. Its estimation in turn is also dependent on the relationship between dose of mediators and change in FEV1. Numerous mediators are involved in asthma, and include histamine and cysteinyl-leukotrienes. There have been studies, albeit few, of urinary leukotriene $\left(\mathrm{LTE}_{4}\right)$ excretion during specific challenges with house dust mite, grass, cat and dog allergens [14, 18]. The excretion of $\mathrm{LTE}_{4}$ during the early asthmatic response was related to the total dose of the inhaled allergen [18]. Surprisingly, although the mean drop in FEV1 was not significantly different after an incremental dose of allergen and after a bolus of the same dose, the excretion of $\mathrm{LTE}_{4}$ during the $2 \mathrm{~h}$ after the cumulative challenge was significantly higher than that after the single bolus [14].

Therefore, the relationship between the early asthmatic response observed during the specific challenge and the concentration, inhaled dose, time and 
deactivation, may be described by a mathematical model, which must also take the specific hyperreactivity of the individual into account.

Highly reactive subjects showed bronchoconstriction immediately after a short exposure to even a low concentration. Their reaction correlated to the inhaled dose. Deactivation could not have prevented the airway obstruction if the lag between exposure and response was short.

Sensitised subjects with intermediate or low hyperreactivity may have asthma symptoms if a sufficient dose is inhaled, that is when the concentration and the duration of the exposure, taking into account deactivation, generate an effective dose higher than the provocative dose for that subject. A given inhaled dose may, if given too slowly or too quickly, be either ineffective or induce bronchoconstriction. Only overexposure during the workshift may effectively cause symptoms. Moreover, a continuous exposure to a low concentration may be ineffective, if the specific reactivity of the subject is low and if the deactivation sufficient [9]. The mathematical model used is able to represent this behaviour adequately: Qc increases with time, concentration, and inspiratory flow rate, but $\mathrm{Qe}$ is limited by an asymptote because of deactivation.

Exposure to a higher concentration increases the slope of the cumulative curve and shifts the asymptote upwards. This finding has implications for prevention measures because limiting the peak concentration would prevent the asthma symptoms in subjects with low reactivity. However, this model may be erroneous if there is a threshold for the bronchial response and if FEV1 does not normalise after the challenge, before a late asthmatic reaction, for example. Analysis of a greater number of subjects is required to confirm or to improve this model. Moreover, several studies have demonstrated that late asthmatic reaction may occur at doses lower than those inducing early reaction [7]. Indeed, repeated inhalation of low doses, without clinical symptoms, increases bronchial hyperresponsiveness and eosinophil cationic protein levels [19, 20]. This continuous exposure without symptoms may also increase the production of antibodies [9].

In summary, the bronchial response to wheat flour depends on the individual's specific hyperreactivity. This specific reactivity varies greatly from one subject to another. The bronchial response was related to the dose of allergen inhaled and to deactivation. This deactivation of the allergen and mediators has to be taken into account if the duration of the specific challenge is extended. A model derived from these observations explains the respective roles of the concentration, of the duration of exposure and of the inhaled dose. The quantification of the dose/response may also have consequences for the prevention of asthma, whatever the type of allergen.

\section{Appendix}

\section{Kinetic model}

If it is assumed that the biological effect induced by the inhaled flour is proportional to the amount of flour in which the allergens remain active in the lung (Qe) and that deactivation of allergenic sites on inhaled flour follows a first order kinetics with a constant $\mathrm{k}$, the differential variation $\mathrm{dQ}$ e during time interval $\mathrm{dt}$ is obtained from a mass balance:

$$
\mathrm{dQ}_{\mathrm{e}}=\mathrm{dQ} \mathrm{i}-\mathrm{kQ} \mathrm{dt}
$$

where $\mathrm{dQ}_{\mathrm{i}}$ is the quantity of flour inhaled during $\mathrm{dt}$; related to aerosol concentration $\mathrm{Ci}$ and inspiratory flow rate $\mathrm{Fi}$ :

$$
\mathrm{dQ}_{\mathrm{i}}=\mathrm{CiFi}_{\mathrm{i}} \mathrm{dt}
$$

Qe can be calculated from the previous inhalation and rest phases by integrating the former differential equation for each phase (inhalation or rest). For a rest phase, $Q$ e decreases from the maximum value obtained at the end of the previous inhalation phase according to an exponential equation. During an inhalation phase, Qe increases exponentially if the flour input dQi/dt is constant, which applies when the aerosol concentration is constant and well controlled. Usually, however, the aerosol concentration fluctuates, but the quantity of flour Qi inhaled over a complete inhalation phase can be calculated accurately by numerical integration of concentration and $\mathrm{Fi}$ [11]. Therefore the differential equation was integrated over each inhalation phase by replacing $\mathrm{dQ} / \mathrm{dt}$ by its mean value, yielding another exponential function. It is noteworthy to emphasise the possibility of calculating Qe for several increasing values of time even after one single inhalation phase, and to plot them versus the corresponding changes in FEV1.

Acknowledgements. The authors would like to thank M.F. Combalot and R. Wrobel for technical assistance.

\section{References}

1. Cartier A, Malo JL. Occupational challenge tests. In: Bernstein IL, Chan-Yeung M, Malo JL, Bernstein DI, eds. Asthma in the Workplace. New York, Marcel Dekker, 1993; pp. 215-247.

2. Kopferschmitt-Kübler MC, Pauli G. Tests de provocation bronchique [Specific bronchial challenge]. In: Bessot JC, Pauli G, ed. L'asthme Professionnel. Paris, Margaux Orange, 1999; pp. 91-108.

3. Choudat D, Fabriès JF, Martin JC, et al. Quantification of the dose of inhaled flour: relation with nonspecific bronchial and immunological reactivities. Eur Respir J 1999; 14: 328-334.

4. Sterk PJ, Fabbri LM, Quanjer PH, et al. Airway responsiveness. Standardized challenge testing with pharmacological, physical and sensitizing stimuli in adults. Eur Respir J 1993; 6: Suppl. 16, 53-83.

5. Bohadana AB, Kopferschmitt-Kübler MC, Pauli G. Breath sound intensity in patients with airway provocation challenge test positive by spirometry but negative for wheezing: a preliminary report. Respiration 1994; 61: 274-279.

6. Taylor DA, Harris JG, $\mathrm{O}^{\prime}$ Connor BJ. Comparison of incremental and bolus dose inhaled allergen challenge in asthmatic patients. Clin Exp Allergy 2000; 30: 56-63. 
7. Vandenplas O, Cartier A, Ghezzo H, Cloutier Y, Malo JL. Response to isocyanates: effect of concentration, duration of exposure, and dose. Am Rev Respir Dis 1993; 147: 1287-1290.

8. Nguyen B, Weytjens K, Cloutier Y, Ghezzo H, Malo JL. Determinants of the bronchial response to high molecular weight occupational agents in a dry aerosol form. Eur Respir J 1998; 12: 885-888.

9. Tiffeneau R. Hypersensibilité cholinergo-histaminique pulmonaire de $1^{\prime}$ asthmatique. Relation avec l'hypersensibilité allergénique pulmonaire [Nonspecific hypersensibility in asthmatic subjects. Reaction with allergic bronchial sensibility]. Acta Allergologica: 1958: Suppl. V, 187-221.

10. Choudat D, Martin JC, Fabriès JF, Villette C, Dessanges JF. Test de provocation bronchique spécifique avec aérosols solides. Quantification des résultats [Specific bronchial challenge with solid particles. Quantification of the results]. Rev Mal Respir 2001; 18: 157-162.

11. Fabriès JF, Choudat $\mathrm{D}$, Wrobel $\mathrm{R}$, et al. Computerized equipment for the delivery of inhaled doses of solid particles in specific bronchial challenge. $J$ Aer Med 2000; 13: 1-10.

12. American Thoracic Society Statement. Standardization of spirometry. 1994 update. Am J Respir Crit Care Med 1995; 152: 1107-1136.

13. Choudat D, Fabriès JF, Martin JC, et al. Modélisation de la relation dose-réponse dans l'asthme à la farine de blé [Dose-response relationship in asthma induced by flour]. Arch Mal Prof 2001; 62: 573.
14. Kumlin M, Dahlen B. The challenge procedure influences the extent of allergen-induced urinary excretion of leukotriene E4. Clin Exp Allergy 2000; 30: 585-589.

15. Merget R, Heger M, Globisch A, et al. Bronchial challenge tests with wheat flour dust administered by Spinhaler: comparison with aqueous wheat flour extract inhalation. J Allergy Clin Immunol 1997; 100: 199-207.

16. Crimi E, Balbo A, Lazzarotti M. Bronchial provocation test with allergen: comparison between two different techniques. Mon Arch Chest Dis 1994; 49: 447-450.

17. Mori L, Kleimberg J, Mancini C, Bellini A, Marini M, Mattoli S. Bronchial epithelial cells of atopic patients with asthma lack the ability to inactivate allergens. Biochem Biophys Res Comm 1995; 217: 817-824.

18. Bancalari L, Conti I, Giannessi D, et al. Early increase in urinary leukotriene E4 (LTE4) is dependent on allergen dose inhaled during bronchial challenge in asthmatic subjects. Allergy 1999; 54: 1278-1285.

19. De Blay F, Krieger P, Spirlet F, et al. Repeated inhalation of low doses of cat allergen that do not induce clinical symptoms increases bronchial hyperresponsiveness and eosinophil cationic protein levels. Int Arch Allergy Immunol 1999; 120: 158-165.

20. Lensmar C, Prieto J, Dahlen B, Eklund A, Grunewald $\mathrm{J}$, Roquet A. Airway inflammation and alveolar macrophage phenotype pattern after repeated lowdose allergen exposure of atopic asthmatic subjects. Clin Exp Allergy 1999; 29: 1632-1640. 